\title{
Influenza: Vacunación a nuevos grupos etarios
}

\author{
Katia Abarca V.
}

\section{Influenza: Immunization to new age groups}

Influenza immunization is a main tool to prevent this important viral illness. Traditional vaccine recommendations, mainly focused to elderly and people with chronic medical conditions, have been expanded during last years. The article summarizes the fundament of lowering the age cut off in adults and of the incorporation of healthy infants between 6 and 24 months. The performance of the vaccine in those new age groups is analyzed. Finally, new groups to possibly be incorporated in future vaccine recommendations are mentioned.

Key words: Influenza; vaccine; children.

Palabras claves: Influenza; vacuna; niños.
Pontificia Universidad Católica

de Chile, Santiago, Chile

Departamento de Pediatría

Recibido: 18 agosto 2005

Aceptado: 22 septiembre 2006

Correspondencia a:

Katia Abarca Villaseca

katia@med.puc.cl
$\mathrm{L}$ a vacunación contra la influenza está recomendada, tradicionalmente, a grupos de riesgo, a saber: adultos sobre 65 años de edad, enfermos crónicos mayores de 6 meses, personal de salud y contactos de sujetos de riesgo. Hace algunos años se incorporaron las mujeres embarazadas.

Más recientemente, en algunos países, se ha ampliado la recomendación de vacunación rutinaria a dos grupos etarios: adultos entre 50 y 65 años (incorporados en E.U.A. desde el año 2000) $)^{1}$ y a niños sanos entre 6 y 24 meses (incorporados en E.U.A. desde el año 2003) ${ }^{2}$. En Chile, se incorporaron los lactantes sanos entre 6 y 24 meses a partir del año 2006 y se redujo la edad de vacunación del adulto a los 60 años, a partir del año 2007.

\section{Vacunación anti-influenza en adultos entre 50 y 65 años}

Este grupo etario posee una elevada prevalencia de condiciones médicas crónicas, la que sido estimada en E.U.A. en $30 \%$. Las tasas de exceso de hospitalización en este grupo son de: 56-635/100.000 en aquellos con una o más condiciones de alto riesgo, comparada con 13-60/100.000 en los que no las presentan ${ }^{3}$. A pesar de tener estas condiciones médicas crónicas, y por tanto estar incluidas desde mucho tiempo en los grupos a vacunar, las coberturas en estas personas efectivamente no eran superiores a $40 \%$. La experiencia ha enseñado que la recomendación de vacunación según grupos etarios es más eficiente que dirigirla hacia las condiciones de riesgo. Es por ello que, a partir del año
2000, el Comité Asesor para la Práctica de Inmunizaciones (Advisory Committee on Immunization PracticesACIP) incorporó a este grupo etario en las recomendaciones de vacuna anual contra influenza ${ }^{1}$. A pesar que esta recomendación implica la vacunación de un grupo importante de personas sin condiciones de riesgo, igualmente ellas tienen importante morbilidad, consultas y ausentismo laboral por influenza, lo que justifica vacunarlas.

La eficacia de la vacuna en adultos entre 50 y 65 años, en condiciones en que la cepa circulante sea similar a la contenida en la vacuna, es de 70 a $90 \%$. Un estudio realizado en la temporada 2003-04 en E.U.A. en que la cepa circulante (H3N2, cepa Fujian) fue diferente de la cepa vaccinal, con 1.055 participantes, 50\% de los cuales poseían condiciones de riesgo $\mathrm{v} / \mathrm{s} 20 \%$ en el grupo controles, se encontró una eficacia de la vacuna (definida como influenza comprobada por laboratorio) de $52 \%$ en las personas sin condiciones de riesgo y de $38 \%$ en aquellas con condiciones de riesgo ${ }^{4}$.

\section{Vacunación de niños}

Tradicionalmente, las recomendaciones de vacunación contra influenza en niños se han focalizado en aquellos con condiciones de riesgo, a partir de los 6 meses de edad (Tabla 1).

Los niños presentan las mayores tasas de ataque de influenza; 15 a $42 \%$ de los pre-escolares y escolares adquieren la infección cada año, comparado con 10 a $20 \%$ en adultos. Las consultas médicas por influenza son elevadas en niños (6-29/100 niños), 3 a 5\% de los 


\section{Tabla 1. Grupos con riesgo elevado de enfermedad grave por virus influenza}

- Asma y enfermedades respiratorias crónicas (fibrosis quística, secuelas graves de neumopatías virales, displasias broncopulmonares, etc)

- Cardiopatías hemodinámicamente significativas

- Inmunocomprometidos, ya sea congénitos o por condiciones adquiridas (infectados con $\mathrm{VIH}$, corticoterapia, quimioterapia, sometidos a trasplantes de órganos sólidos y de precursores hematopoyéticos, etc)

- Hemoglobinopatías

- Enfermedad renal crónica

- Enfermedades metabólicas (diabetes y otras)

- Usuarios crónicos de ácido acetilsalicílico niños tienen anualmente otitis media aguda (OMA) asociada a influenza. La influenza y sus complicaciones producen un aumento de 10 a $30 \%$ en el uso de antimicrobianos. La infección se asocia con neumonía por Streptococcus pneumoniae y Staphylococcus aureus. Las tasas de hospitalización bajo 5 años de edad son de 1/1.000 en ausencia de condiciones de riesgo, y de 5/1.000 con condiciones de riesgo.

Por todo ello, la tendencia de los últimos años ha sido incorporar en la vacunación anti-influenza a otros grupos de niños: lactantes sanos entre 6 y 24 meses de edad, los contactos cercanos bajo 2 años de edad, niños con OMA recurrente o con infecciones respiratorias recurrentes, niños sanos que asisten a centros de cuidado, e incluso escolares.

Niños sanos entre 6 y 24 meses. Durante los últimos años, en que se ha podido establecer con más certeza la etiología viral de las infecciones respiratorias que motivan internación en niños, se ha demostrado que las tasas de hospitalización por influenza bajo 2 años de edad son elevadas (190-480/100.000) y tan altas o mayores que las observadas en adultos sobre 65 años de edad (125-218/100.000) ${ }^{5}$.

Diversos estudios han mostrado el impacto de la hospitalización por influenza en niños sanos: en el Hospital de Niños de Toronto, Montreal, en el año 1999-2000 se internaron 182 niños por influenza, 66\% tenían sobre 6 meses de edad, $70 \%$ de ellos no tenían enfermedades crónicas, en $31 \%$ de ellos el diagnóstico de ingreso fue sepsis ${ }^{6}$. Dos estudios chilenos han mostrado similares resultados: en el Hospital Clínico de la Pontificia Universidad Católica de Chile, durante los años 2001 y 2004 se hospitalizaron 91 niños por influenza; 68 y $81 \%$ de ellos tenía bajo 2 años de edad, 45 y $80 \%$ no tenían enfermedades crónicas (Perret y cols. XXIV Congreso Chileno Infectología 2004). En el Hospital San Borja Arriarán, el año 1999 ingresaron 177 niños por influenza, $80 \%$ tenían bajo 2 años de edad, $76 \%$ de ellos no padecían de enfermedades crónicas ${ }^{7}$. Estos datos confirman que la mayoría de los niños hospitalizados por influenza son lactantes sin patología de base.

Es por esto, que a partir del año 2000, la Academia Americana de Pediatría, por intermedio del ACIP, incorporó en su recomendación la vacunación rutinaria anti-influenza a lactantes sanos entre 6 y 24 meses de $\operatorname{edad}^{2}$.

\section{Comportamiento de la vacuna inactivada en niños}

Reactogenicidad: Se han observado síntomas locales en 3 a $27 \%$ de los niños vacunados (dolor, eritema, edema en el sitio de inyección), síntomas sistémicos en 4 a $16 \%$, siendo los más frecuentes fiebre y coriza, fiebre en 10 a $35 \%$ (más frecuente bajo 2 años de edad), sin diferencias con el grupo placebo. Otros síntomas reportados, generalmente leves, son: náuseas, letargia, cefalea, mialgias, calofríos. Generalmente, los eventos no han mostrado diferencias entre vacunados y quienes recibieron placebo, entre mayores y menores de 12 meses, con excepción de la fiebre, y no aumentan con la administración de dosis sucesivas ${ }^{8-12}$.

Seguridad: En 250.000 niños de 6 meses a 17 años, no se observó aumento de eventos que requirieran atención médica ${ }^{13}$. La vacunación no aumenta la hiperreactividad bronquial en asmáticos ${ }^{14}$; no se han observado eventos adversos serios en más de 250.000 niños bajo 18 años de edad y más de 430.000 dosis de vacu$\mathrm{na}^{15}$.

Un estudio focalizado en niños bajo 2 años de edad, utilizando los datos del sistema de vigilancia de eventos adversos a vacunas de E.U.A., (Vaccine Adverse Events Report System- VAERS), reportó 166 eventos adversos en estos niños durante los años 1990 a 2003; de ellos, 37\% recibió sólo vacuna anti-influenza, el resto la recibió en conjunto con otra vacuna. Los eventos adversos más frecuentes fueron: fiebre (59: $35 \%$ ), rash no especificado o urticarial (42: $25 \%$ ), convulsiones (28: $17 \%$ ) y reacción en el sitio de la inyección (28: 17\%). El $68 \%$ de las convulsiones fueron febriles y ocurrieron dentro de las 48 horas post vacunación. No se observaron cambios importantes en este sistema de registro después de iniciar la vacunación rutinaria de niños entre 6 y 24 meses en E.U.A. Este estudio concluyó que la vacuna tiene un buen perfil de seguridad en lactantes, pero sugirió mantener vigilancia de la incidencia de convulsiones ${ }^{16}$.

El síndrome de Guillain Barré se ha observado en relación a la vacuna anti-influenza utilizada en adultos en la formulación del año 1976 ( 1 caso por millón de vacunados). En niños de 6 a 24 meses, no se ha 
observado esta asociación con enfermedad desmielinizante. El síndrome oculo-respiratorio observado excepcionalmente en adultos (ojos rojos, edema facial, síntomas respiratorios, disfagia, odinofagia), leve y transitorio, no ha sido descrito en pediatría. En la vacunación en niños con infección por VIH se ha observado un aumento transitorio de replicación del VIH (carga viral) y/o baja en los niveles de CD4, pero sin alteraciones clínicas, por lo que los expertos indican que los beneficios de vacunar superan los riesgos de afectar el curso de la infección por VIH.

\section{Contraindicaciones de la vacuna anti-influenza en niños}

Está contraindicada formalmente en:

- Lactantes bajo 6 meses de edad.

- Reacción anafiláctica (cutánea, respiratoria, colapso cardiovascular) desencadenada por el consumo de pollo o proteínas de huevo.

- Reacción anafiláctica a otros componentes de la vacuna (como el timerosal).

\section{Eficacia de la vacuna en niños}

Un meta-análisis de 13 estudios randomizados, mostró las siguientes cifras de eficacia: contra influenza confirmada por cultivo: $74 \%$ (57-84), influenza confirmada por serología: 59\% (43-71), enfermedad clínica: $33 \%$ (29-36). No hubo diferencias entre el empleo de vacunas inactivadas y vivas atenuadas, y se observó una tendencia a aumentar la eficacia con la edad ${ }^{17,18}$.

La eficacia también ha sido estudiada en lactantes de 6 a 24 meses. En la temporada 2003-04, en Colorado, E.U.A., en que la cepa circulante fue diferente de la cepa vaccinal, mediante un estudio de cohorte, retrospectivo, que comprendió 5.139 niños, 7\% con enfermedades crónicas, $20 \%$ vacunados antes de la estación (dos dosis), se demostró una eficacia ( $\sin$ confirmación de laboratorio), de $25 \%$ para enfermedad tipo influenza y $49 \%$ para neumonía e influenza. En los niños parcialmente vacunados no se observó una reducción significativa de la infección respecto a los no vacunados ${ }^{4}$.

Otro interesante estudio examinó cuáles eran los factores determinantes para que un lactante recibiera la vacuna; el factor más importante encontrado fue la recomendación de vacunación por parte del pediatra: si el pediatra recomendaba la vacuna, el 70\% la recibió, mientras que si el pediatra no la recomendaba sólo $3 \%$ la recibió ${ }^{19}$.

El grado de evidencia para recomendar de vacunación contra influenza en niños se detalla en la Tabla 2 .

\section{Tabla 2. Grado de evidencia para recomendar la vacunación en niños}

\begin{tabular}{|c|c|c|}
\hline & - Niños alto riesgo (enfermedades crónicas) & Evidencia $\|-3$ \\
\hline & - Contactos de niños de alto riesgo & Evidencia $\|-3$ \\
\hline & - Niños sanos entre 6 y 24 meses & Evidencia $\|-3$ \\
\hline & - Familiares y cuidadores de niños $<24$ meses & Evidencia III \\
\hline \multicolumn{3}{|c|}{ Grados de evidencia: } \\
\hline । & \multicolumn{2}{|l|}{ Estudios controlados randomizados } \\
\hline$\|-1$ & \multicolumn{2}{|c|}{ Estudios bien diseñados no randomizados } \\
\hline $11-2$ & \multicolumn{2}{|c|}{ Estudios de cohortes o caso control bien diseñados en más de un centro } \\
\hline $11-3$ & \multicolumn{2}{|c|}{$\begin{array}{l}\text { Múltiples series con o sin intervención, o dramáticos resultados en estudios no contro- } \\
\text { lados }\end{array}$} \\
\hline III & \multicolumn{2}{|c|}{$\begin{array}{l}\text { Opiniones de autoridades respetadas, basados en experiencia clínica, estudios descripti- } \\
\text { vos, reportes de expertos }\end{array}$} \\
\hline
\end{tabular}

\section{Otras recomendaciones de vacunación anti- influenza a niños}

- Niños con OMA recurrente: Algunos estudios han mostrado reducción en la incidencia de OMA que alcanza al $36 \%$ en niños vacunados ${ }^{20}$, riesgo 0,69 en vacunados $(0,49-0,98)^{21}$; en cambio, otro estudio no mostró esta reducción ${ }^{22}$.

- Niños con infecciones recurrentes del tracto respiratorio.

- Niños sanos que asisten a centros de cuidado.

- Escolares.

\section{Vacunación anti-influenza en escolares}

Diversos autores han resaltado la eficacia de vacunar escolares. Vacunar 70 a $80 \%$ de los escolares tendría el mismo efecto que vacunar 185 millones de personas de alto riesgo y sus contactos ${ }^{23}$. Vacunar niños entre 6 meses y 18 años, con una cobertura de $20 \%$, reduciría el número de casos de influenza en $46 \%$; con una cobertura de $80 \%$ la reducción sería de $91 \%$, con similares reducciones en muertes y costos económi$\cos ^{24}$. Vacunar a $20 \%$ de los niños de 18 meses a 18 años, otorgaría protección indirecta a los adultos sobre 35 años, con una reducción de consultas médicas por enfermedad respiratoria aguda de 8 a $18 \%{ }^{25}$. En Japón, la vacunación de escolares evitó 37.000 a 49.000 muertes, o 1 muerte por cada 420 niños vacunados ${ }^{26}$.

La política de vacunar escolares sanos parece ser costo-efectiva. La ACIP, en el año 2003, estimó que si el costo de vacunar es menor de US \$30 por niño, se genera un ahorro desde el punto de vista social ${ }^{27}$.

Dentro de las estrategias para lograr mejores coberturas de vacunación anti-influenza a niños están:

- Recomendación entregada por el pediatra.

- Vacunación en grupos (campañas).

- Vacunación disponible fuera del horario de trabajo de los padres.

- Cobertura por seguros. 


\section{Resumen}

La vacunación anti-influenza es la herramienta fundamental para la prevención de esta importante enfermedad viral. Las indicaciones de vacunación, tradicionalmente enfocadas a los adultos a partir de los 65 años y a portadores de enfermedades crónicas, se han ido ampliando en los últimos años. Se presenta el fundamento de la reducción de la edad de vacunación de adultos y de la incorporación de lactantes sanos entre 6 y 24 meses de edad y se analiza el comportamiento de la vacuna en estos grupos etarios. Finalmente, se indican nuevos grupos posibles a incorporar en los programas de vacunación en el futuro.

\section{Referencias}

1.- Bridges C B, Winquist A G, Fukuda K, Cox N J, Singleton J A, Strikas R A; Advisory Committee on Immunization Practices. Prevention and control of influenza: recommendations of the Advisory Committee on Immunization Practices (ACIP). MMWR Recomm Rep 2000; 49 (RR-3): 1-38.

2.- Bridges C B, Harper S A, Fukuda K, Uyeki T M, Cox N J, Singleton J A; Advisory Committee on Immunization Practices. Prevention and control of influenza. Recommendations of the Advisory Committee on Immunization Practices (ACIP). MMWR Recomm Rep 2003; 52 (RR-8): 1-34.

3.- Harper S A, Fukuda K, Uyeki T M, Cox N J, Bridges C B; Centers for Disease Control and Prevention (CDC) Advisory Committee on Immunization Practices (ACIP). Prevention and control of influenza: recommendations of the Advisory Committee on Immunization Practices (ACIP). MMWR Recomm Rep 2004; 53 (RR-6): 1-40.

4.- Centers for Disease Control and Prevention. Assessment of the effectiveness of the 2003-04 influenza vaccine among children and adults. Colorado, 2003. MMWR Morbid Mortal Wkly Rep 2004; 53: 707-10.

5.- Izurieta H S, Thompson W W, Kramarz P, Shay D K, Davis R L, DeStefano F, et al. Influenza and the rates of hospitalization for respiratory disease among infants and young children. N Engl J Med 2000; 342: 232-9.

6.- Quach C, Piche-Walker L, Platt R,

Moore D. Risk factors associated with severe influenza infections in childhood: implication for vaccine strategy. Pediatrics. 2003; 112: e197-201.

7.- Delpiano L, Byron G, Casado C. Comportamiento clínico-epidemiológico de la influenza en niños hospitalizados. Rev Chil Infectol 2003; 20: 159-65.

8.- Gruber W C, Taber L H, Glezen W P, Clover R D, Abell T D, Demmler R W, et al. Live attenuated and inactivated influenza vaccine in school-age children. Am J Dis Child 1990; 144 (5): 595-600.

9.- Piedra P A, Glezen W P, Mbawuike I, Gruber W C, Baxter B D, Boland F J, et al.
Studies on reactogenicity and immunogenicity of attenuated bivalent cold recombinant influenza type A (CRA) and inactivated trivalent influenza virus (TI) vaccines in infants and young children. Vaccine 1993; 11: 718-24.

10.- Khan A S, Polezhaev F, Vasiljeva R, Drinevsky V, Buffington J, Gary H, et al. Comparison of US inactivated split-virus and Russian live attenuated, cold-adapted trivalent influenza vaccines in Russian school children. J Infect Dis 1996; 173: 453-6.

11.- Neuzil K M, Dupont W D, Wright P F, Edwards K M. Efficacy of inactivated and cold-adapted vaccines against influenza A infection, 1985 to 1990: the pediatric experience. Pediatr Infect Dis J 2001; 20: 733-40.

12.- Edwards K M, Dupont W D, Westrich M K, Plummer W D Jr, Palmer P S, Wright P F. A randomized controlled trial of coldadapted and inactivated vaccines for the prevention of influenza A disease. J Infect Dis 1994; 169: 68-76.

13.- France E K, Glanz J M, Xu S, Davis R L, Black S B, Shinefield H R, et al. Safety of the trivalent inactivated influenza vaccine among children: a population-based study. Arch Pediatr Adolesc Med 2004; 158: 10316.

14.- Kramarz P, Destefano F, Gargiullo P M, Chen R T, Lieu TA, Davis RL, et al; Vaccine Safety Datalink Team. Does influenza vaccination prevent asthma exacerbations in children? J Pediatr 2001; 138: 306-10.

15.- Chen R T, DeStefano F, Davis R L, Jackson L A, Thompson R S, Mullooly J P, et al. The Vaccine Safety Datalink: immunization research in health maintenance organizations in the USA. Bull World Health Org 2000; 78: 186-94.

16.- McMahon A W, Iskander J, Haber P, Chang S, Woo E J, Braun M M, et al. Adverse events after inactivated influenza vaccination among children less than 2 years of age: analysis of reports from the vaccine adverse event reporting system, 1990-2003. Pediatrics 2005; 115: 453-60.

17.- Negri E, Colombo C, Giordano L, Groth N, Apolone G, La Vecchia C. Influenza vaccine in healthy children: a meta-analysis. Vaccine
2005; 23: 1851-61.

18.- Zangwill K M, Belshe R B. Safety and efficacy of trivalent inactivated influenza vaccine in young children: a summary for the new era of routine vaccination. Pediatr Infect Dis J 2004; 23: 189-200.

19.- Poehling K A, Speroff T, Dittus R S, Griffin M R, Hickson G B, Edwards K M. Predictors of influenza virus vaccination status in hospitalized children. Pediatrics 2001; 108: e99.

20.- Heikkinen T, Ruuskanen O, Waris M, Zieger T, Arola M, Halonen P. Influenza vaccination in the prevention of otitis media in children. AJDC 1991; 145: 445-8.

21.- Clements D A, Langdon L, Bland C, Emmanuel W. Influenza vaccine decreases incidence of otitis media in 6-30 month-old children in day care. Arch Pediatr Adolesc Med 1995: 149; 1113-7.

22.- Hoberman A, Greenberg D P, Pardise J L, Rockette H E, Lave J R, Keraney D et al. Effectiveness of inactivated influenza vaccine in preventing acute otitis media in young children. JAMA 2003; 290: 1608-16.

23.- Longini I M, Halloran M E, Nizam A, Wolff M, Mendelman P M, Fast P E, et al. Estimation of the efficacy of live, attenuated influenza vaccine from a twoyear, multi-center vaccine trial: implications for influenza epidemic control. Vaccine 2000 Mar 17; 18: 1902-9.

24.- Weycker D, Edelsberg J, Halloran M E, Longini I M, Nizam A, Ciuryla V, et al. Population-wide benefits of routine vaccination of children against influenza. Vaccine 2005; 23: 1284-93.

25.- Piedra P A, Gaglani M J, Kozinetz C A, Herschler G, Riggs M, Griffith M, et al. Herd immunity in adults against influenza-related illnesses with use of the trivalent-live attenuated influenza vaccine (CAIV-T) in children. Vaccine 2005; 23: 1540-8.

26.- Reichert T A, Sugaya N, Fedson D S, Glezen W P, Simonsen L, Tashiro M. The Japanese experience with vaccinating schoolchildren against influenza. N Engl J Med 2001; 344: 889-96.

27.- White T, Lavoie S, Nettleman M D. Potential cost savings attributable to influenza vaccination in school-aged children. Pediatrics 1999; 103: e73. 